BMJ Open Sport \& Exercise Medicine

\title{
Effect of pseudoephedrine in sport: a systematic review
}

\author{
Kien V Trinh, ${ }^{1}$ Jiin Kim, ${ }^{2}$ Amanda Ritsma ${ }^{1}$
}

To cite: Trinh KV, Kim J, Ritsma A. Effect of pseudoephedrine in sport: a systematic review. BMJ Open Sport Exerc Med 2015;1:e00066. doi:10.1136/bmjsem-2015000066

- Prepublication history and additional material is available. To view please visit the journal (http://dx.doi.org/ 10.1136/bmjsem-2015000066).

Accepted 18 November 2015

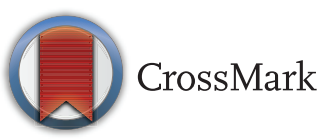

${ }^{1}$ Faculty of Medicine, McMaster University, Hamilton, Ontario, Canada ${ }^{2}$ Faculty of Health Sciences, McMaster University, Hamilton, Ontario, Canada

Correspondence to Dr Kien V Trinh; trinhk@mcmaster.ca

\section{ABSTRACT}

Objective: Pseudoephedrine is a stimulant that can be purchased over-the-counter to relieve symptoms of nasal and sinus congestion. Owing to its similar composition to ephedrine and other amphetamines, pseudoephedrine mirrors some of its ergogenic effects. This study investigates its possible ergogenic effect through a systematic review. Our primary aim was to determine the effects of pseudoephedrine in sport and its potential for performance enhancement. Design: We searched EMBASE, MEDLINE, PsychINFO and The Cochrane Library for trials conducted from their beginning to March 2015. Any published trial that used randomised assignment to the intervention and control groups in full text and measured pseudoephedrine as an independent variable were included.

Results: Overall, the review showed that the ergogenic effect of pseudoephedrine is dose-dependent. None of the reviewed studies showed an ergogenic effect at the therapeutic dose of the drug (60-120 mg); however, supratherapeutic doses $(\geq 180 \mathrm{mg})$ yielded clinically significant results.

Conclusions: Owing to the limitations of the published studies in this field, we were unable to make any firm conclusions with respect to the overall effect of pseudoephedrine and its ergogenic effect. It is evident that there is a correlation between the dose administered and its ergogenic effects, but it is also evident that the side effects of using above the therapeutic dose outweigh the possible benefits of using pseudoephedrine in sport. Further research with larger sample sizes is required to determine the relationship between doses $(\geq 180 \mathrm{mg}$ ) and concentrations in urine that cause an ergogenic effect.

\section{BACKGROUND}

During the 1995 Pan American Games, Silken Laumann sailed to victory with her teammates in the women's quadruple event. ${ }^{1}$ Five days later, Laumann's drug screen showed levels of the prohibited stimulant pseudoephedrine (PSE), and the gold medal was revoked. It was later determined that Laumann had inadvertently taken over-the-counter PSE-containing medication for symptomatic relief of her cold. ${ }^{1}$ Jack Uetrecht, a professor of pharmacy and medicine at the University of Toronto claimed that the dose and form Laumann took 'did not enhance performance', the officials of the games did not agree. ${ }^{1}$ The banning of PSE has since become a highly debated topic in both the athletic and medical field.

PSE is a sympathomimetic amine that is readily available over-the-counter as a nasal and sinus decongestant. ${ }^{2}$ Specifically, PSE activates adrenergic receptors in presynaptic neurons, which causes vasoconstriction. This decreases inflammation and mucous production $^{2}$ which relieves symptoms of the common cold. PSE has also been proposed to have ergogenic effects, likely due to its similarity to ephedrine and other central nervous system stimulants. These effects include increased muscle contractility, increased blood flow to skeletal muscles, increased glycogenesis, increased central nervous activation and heart rate, as well as decreased time to fatigue. ${ }^{3}$ The International Olympic Committee and other organisations have banned the use of any substance that may enhance the sympathetic nervous system and, by its nature, have concluded that PSE has the potential to have this effect. Owing to the ergogenic nature of this drug, it is believed that it is a violation of the spirit of sport. Therefore, PSE was banned from use in competition.

It has been debated whether or not PSE is actually capable of generating any ergogenic effect. This continued debate has resulted in multiple changes to its position on the prohibited and/or monitoring list. Until 2004, PSE was included on the International Olympic Committee prohibited list. From 2004 to 2010, PSE was removed from the prohibited list, and later added to the monitoring list for in competition in $2010 .^{4}$ In this position, the use of PSE was considered doping if the urine concentration was greater than $150 \mu \mathrm{g}$ while in competition. ${ }^{4}$ Recently, as of 1 January 2015, PSE has been removed from the monitoring list. ${ }^{4}$

Despite potential risks and uncertainty on the ergogenic effects of PSE, athletes have 
still been known to abuse PSE for its potential enhancement ability. Data collected by the World Anti Doping Association between 1996 and 2003 yielded 33 adverse analytical findings for PSE out of 52347 in-competition analyses, or 4.1 positive controls per year. ${ }^{45}$ In 2007 and 2008 , that is, 3 years after PSE was removed from the prohibited list, the prevalence of PSE and ephedrine was determined in 16335 in competition doping control samples. ${ }^{45}$ The analyses resulted in 102 cases of PSE use or misuse. ${ }^{45}$

The purpose of this systematic review is to qualitatively consolidate the results of studies relating to the ergogenic effect of PSE in order to determine the validity of its ban from competition. Previous studies have yet to resolve the existing conflicting results, even when standardised testing methods are utilised. ${ }^{6-8}$ Therefore, this article aims to clarify the relationship between PSE and sport enhancement on various parameters, specifically relating to drug dosage.

\section{OBJECTIVES}

To determine the effects of PSE in sport and its potential for performance enhancement.

\section{CRITERIA FOR CONSIDERING STUDIES FOR THIS REVIEW Types of studies}

Any published randomised control trial (RCT) in the English language, including cross-over studies. Owing to the controversy in this area, the authors felt that randomised controlled studies were the most appropriate research design to minimise bias to address the effectiveness of intervention. Studies were excluded if PSE was not the sole substance being administered to an athlete at a given time, or if the substance was not specifically being investigated for its ergogenic effects. This limitation was to ensure the data presented were not affected by any confounding variables.

\section{Types of participants}

Participants were male and female athletes of any level between age 18 and 65, with no other comorbid conditions.

\section{Types of interventions}

Studies must have used PSE as the only substance in the intervention. Studies that looked at other substances were included if athletes were not administered both substances simultaneously. The presence of a control and/or placebo group was also necessary for inclusion.

\section{Types of outcome measures}

Outcomes measured included any enhancement in sport above baseline such as timing, strength, time to fatigue and/or respiratory enhancement.

\section{METHODS}

\section{Search strategy}

We searched EMBASE, MEDLINE, PsycInfo and Cochrane Library databases for trials from their beginning to March 2015 (figure 1).

\section{Study selection}

At least two authors independently conducted citation identification, study selection and data abstraction. Disagreements were resolved through a third assessor.

\section{Methodological assessment}

At least two authors independently assessed each RCT for methodological quality and bias, based on the Cochrane's GRADE scale and the Cochrane's collaboration tool for assessing risk of bias. ${ }^{9} 10$ Disagreements were resolved through a third assessor.

\section{Data extraction}

Two authors independently extracted raw data for demographics, descriptions of interventions and all outcomes to predesigned forms.

\section{Data analysis}

Data were retrieved and filed into abstraction forms. Differences between assessors were resolved by repeated review and consensus. The risk of bias of the RCT was assessed through the use the Cochrane collaboration's tool for assessing risk of bias. A third assessor resolved differences between assessors.

\section{DESCRIPTION OF STUDIES/STUDY SELECTION}

In consultation with two research librarians, we developed search strategies to identify potentially relevant studies from the EMBASE, MEDLINE, PsycInfo and Cochrane Library databases (see online supplementary appendix 1). We sought reports of RCTs, including cross-over trials, in relation to PSE use for its ergogenic effect. Clinical judgement was used to review the search and retrieve potentially relevant studies. Studies were excluded if they had co-interventions with other drugs (table 1).

\section{METHODOLOGICAL QUALITY}

Methodological quality was graded using two sets of criteria:

- Risk of bias: based on selection, performance, detection, attrition, reporting and other biases. ${ }^{10}$

- Cochrane GRADE table: began with highest quality rating for randomised trial evidence with downgrades to moderate, low or very low depending on the presence of limitations in design, indirectness of evidence, inconsistency of results, imprecision of results and high probability of publication bias. ${ }^{9}$ 
Figure 1 PRISMA 2009 flow diagram.

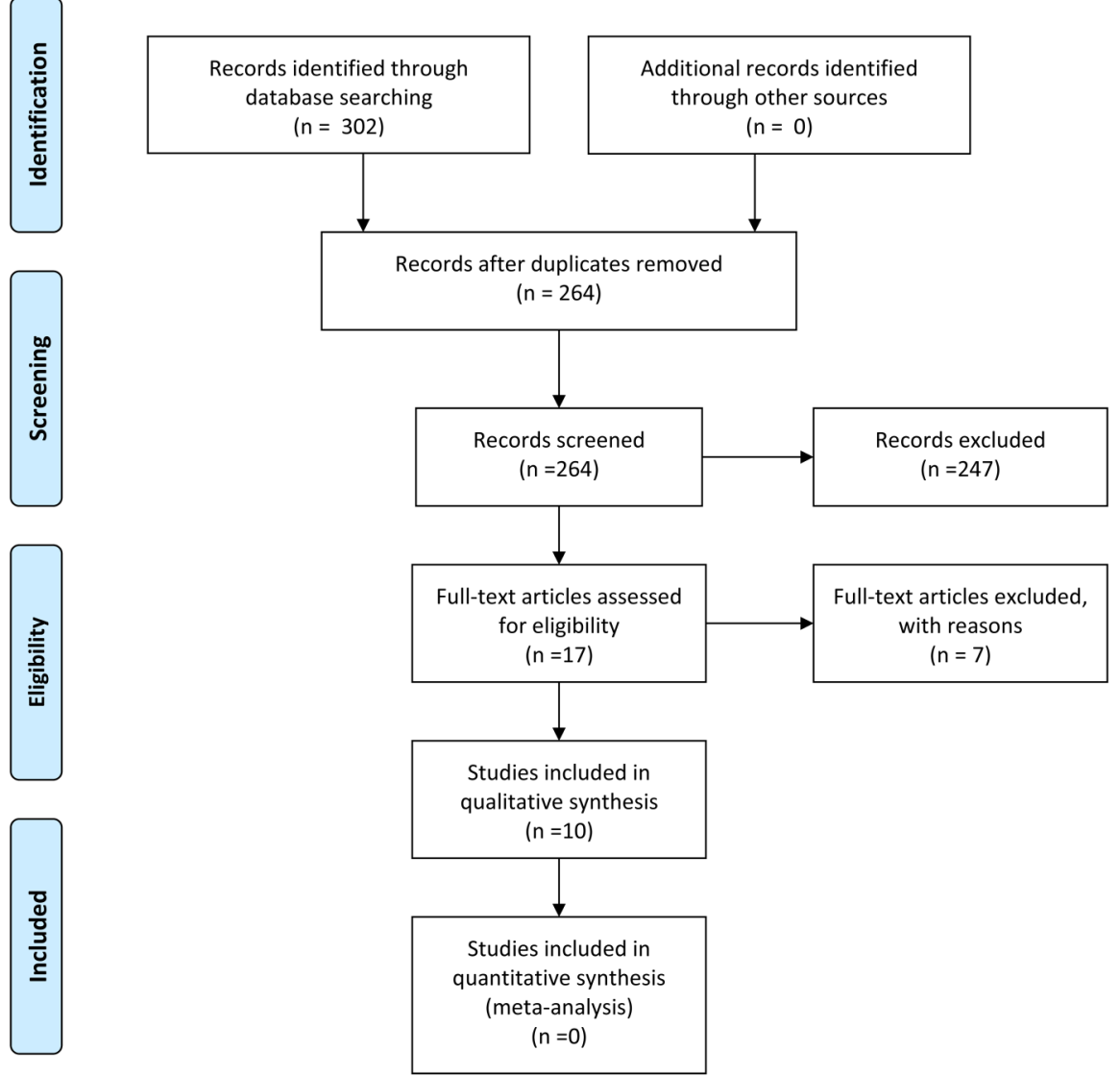

\section{RESULTS}

Out of 301 articles retrieved from EMBASE, MEDLINE, PsycInfo and The Cochrane Library, 39 duplicates were removed. From the 262 remaining, 17 studied the ergogenic effects of PSE and PSE-like substances. Of these 17 , only 10 were devoted solely to studying the ergogenic effects of PSE and were used for this systematic review. Therefore, 10 remaining studies met all inclusion criteria (table 2).

\begin{tabular}{|c|c|}
\hline Study & Reason for exclusion \\
\hline Barroso et al ${ }^{11}$ & $\begin{array}{l}\text { Focuses on urinary threshold for } \\
\text { detection of PSE }\end{array}$ \\
\hline Bell and Jacobs ${ }^{12}$ & $\begin{array}{l}\text { Combined effect of caffeine and } \\
\text { PSE studied }\end{array}$ \\
\hline Bell et $a l^{13}$ & $\begin{array}{l}\text { Combined effect of caffeine and } \\
\text { PSE studied }\end{array}$ \\
\hline Chester et al ${ }^{14}$ & $\begin{array}{l}\text { Focuses on urinary threshold for } \\
\text { detection of PSE }\end{array}$ \\
\hline Jolley et al ${ }^{15}$ & $\begin{array}{l}\text { Focuses on influence of dehydration } \\
\text { on PSE urinary levels }\end{array}$ \\
\hline Pokrywka et al ${ }^{16}$ & Focuses on frequency of PSE use \\
\hline Spence et $a l^{17}$ & $\begin{array}{l}\text { Focuses on differences between } \\
\text { effects of caffeine and PSE }\end{array}$ \\
\hline
\end{tabular}

\section{Placebo versus PSE}

Of the 10 chosen studies, 3 showed significant improvement in athletic performance. ${ }^{2024} 25$ These three studies used a supratherapeutic dose of at least $180 \mathrm{mg}$ or $2.5 \mathrm{mg} / \mathrm{kg}$ of PSE, whereas the other studies used a therapeutic dose $(60-120 \mathrm{mg}$ or $1-2 \mathrm{mg} / \mathrm{kg})$. The studies that used lower dosage of PSE showed no significant improvement for all measured parameters. ${ }^{18} 1921-242627$ The authors compared both $60-120 \mathrm{mg}$ or $1-2 \mathrm{mg} / \mathrm{kg}$ and $\geq 180 \mathrm{mg}$ or $2.5 \mathrm{mg} / \mathrm{kg}$ of PSE to a placebo of similar appearance on the effects on maximal torque and peak power, decreased time to complete trial, and respiratory function. While the placebo and $60-120 \mathrm{mg}$ or $1-2 \mathrm{mg} /$ $\mathrm{kg}$ PSE categories showed no significant change, the $\geq 180 \mathrm{mg}$ or $2.5 \mathrm{mg} / \mathrm{kg}$ PSE group showed significant improvement for all measured parameters. However, due to heterogeneity of the measurements selected in the trials, quantitative synthesis of data was not possible.

\section{DISCUSSION}

Our objective was to determine the effects of PSE on athletic performance in athletic individuals in good general health. Qualitative analysis showed overall positive results in favour of PSE over placebo for PSE doses $\geq 180 \mathrm{mg}$ or $2.5 \mathrm{mg} / \mathrm{kg}$. Doses below $180 \mathrm{mg}$ or $2.5 \mathrm{mg} / \mathrm{kg}$ and placebos showed insignificant changes in athletic performance.

Interventions varied with respect of duration of treatment, doses of PSE, diet and type of exercise trial. For 
Table 2 Characteristics of included studies

\begin{tabular}{|c|c|c|c|c|c|}
\hline Study & Design & Subject description & PSE dosage & Main Interventions & Outcome results \\
\hline$\overline{\text { Gillies et } a l^{18}}$ & $\begin{array}{l}\text { Double-blind RCT } \\
\text { cross-over, single } \\
\text { dose }\end{array}$ & $\begin{array}{l}\text { Volunteer sample of } 10 \text { healthy } \\
\text { male cyclists with no history of } \\
\text { renal or other diseases }\end{array}$ & $\begin{array}{l}120 \mathrm{mg} 90 \mathrm{~min} \\
\text { prior to testing }\end{array}$ & $\begin{array}{l}\text { Group A ( } n=10) \\
120 \mathrm{mg} \text { PSE or placebo with } \\
\text { exercise, } 1 \text {-week washout period } \\
\text { (2 trials) } \\
120 \text { PSE or placebo with no } \\
\text { exercise (1 trial) } \\
\text { Exercise: isometric muscle test } \\
\text { (peak value) followed by } 40 \mathrm{~km} \\
\text { cycling trial (time to completion) }\end{array}$ & $\begin{array}{l}\text { No significant improvement in } \\
\text { any parameters (isometric } \\
\text { muscle function) }\end{array}$ \\
\hline Swain et a $\left.\right|^{19}$ & $\begin{array}{l}\text { Double-blind RCT, } \\
\text { multiple-dose }\end{array}$ & $\begin{array}{l}\text { Convenience sample of } 20 \text { male } \\
\text { cyclists ( } 18-35 \text { ), cycling } 50+ \\
\text { miles a week; } 10 \text { relevant to PSE }\end{array}$ & $\begin{array}{l}1 \mathrm{mg} / \mathrm{kg}, 2 \mathrm{mg} / \\
\mathrm{kg} 60 \mathrm{~min} \text { prior } \\
\text { to testing }\end{array}$ & $\begin{array}{l}\text { Group A }(n=10) \\
1 \mathrm{mg} / \mathrm{kg}, 2 \mathrm{mg} / \mathrm{kg} \text { or placebo with } \\
\text { exercise, } 1 \text {-week washout period } \\
\text { ( } 3 \text { trials) } \\
\text { Exercise: bicycle ergometer tests } \\
\text { (time to exhaustion, } \mathrm{VO}_{2 \text { max }} \text {, peak } \\
\text { pulse, and RPE) }\end{array}$ & $\begin{array}{l}\text { No significant improvement in } \\
\text { any parameters }\left(\mathrm{VO}_{2 \max } \text {, time }\right. \\
\text { to exhaustion, peak } \mathrm{BP} \text { and } \\
\text { pulse) for either dose }\end{array}$ \\
\hline Gill et $a R^{0}$ & $\begin{array}{l}\text { RCT cross-over, } \\
\text { single dose }\end{array}$ & $\begin{array}{l}\text { Volunteer sample of } 22 \text { healthy } \\
\text { male athletes from university } \\
\text { student population with no } \\
\text { reported injuries prior to study }\end{array}$ & $\begin{array}{l}180 \mathrm{mg} 45 \mathrm{~min} \\
\text { prior to testing }\end{array}$ & $\begin{array}{l}\text { Group A ( } n=22) \\
180 \text { mg PSE with exercise, } 1 \text {-week } \\
\text { washout period ( } 1 \text { trial) } \\
\text { Placebo with exercise ( } 1 \text { trial) } \\
\text { Exercise: Wingate test, isometric } \\
\text { leg extension, bench press (peak } \\
\text { value, heart rate) }\end{array}$ & $\begin{array}{l}\text { Improved maximum torque, } \\
\text { improved peak power of } \\
\text { maximal cycling, improved } \\
\text { respiratory function }\end{array}$ \\
\hline Chester et $a$ f $^{1}$ & $\begin{array}{l}\text { RCT cross-over, } \\
\text { multiple dose }\end{array}$ & $\begin{array}{l}\text { Recruited sample of } 8 \text { male } \\
\text { endurance runners }\end{array}$ & $\begin{array}{l}60 \mathrm{mg}, 6 \text { doses } \\
\text { over } 36 \mathrm{~h}, 4 \mathrm{~h} \\
\text { prior to testing }\end{array}$ & $\begin{array}{l}\text { Group A ( } n=8): 60 \mathrm{mg} \text { PSE or } \\
\text { placebo with exercise, } 1 \text {-week } \\
\text { washout period ( } 4 \text { trials) } \\
\text { Exercise: steady state exercise, } \\
5000 \mathrm{~m} \text { time trial }\left(\mathrm{VO}_{2 \max } \text {, heart }\right. \\
\text { rate, BP, peak time) } \\
\text { Statistical data not reported }\end{array}$ & $\begin{array}{l}\text { No significant improvement in } \\
\text { any parameters }\left(\mathrm{VO}_{2 \max } \text {, heart }\right. \\
\text { rate, and respiratory exchange } \\
\text { ratio) }\end{array}$ \\
\hline Chu et $a$ P2 $^{2}$ & $\begin{array}{l}\text { Double-blind RCT } \\
\text { cross-over, single } \\
\text { dose }\end{array}$ & $\begin{array}{l}\text { Volunteer sample of } 10 \text { male, } 9 \\
\text { female healthy university students } \\
\text { (1 dropout) }\end{array}$ & $\begin{array}{l}120 \mathrm{mg}, 2 \mathrm{~h} \\
\text { prior to testing }\end{array}$ & $\begin{array}{l}\text { Group A ( } n=19 \text { ) } \\
120 \text { mg PSE or placebo with } \\
\text { exercise, } 1 \text {-week washout period } \\
\text { ( } 2 \text { trials) } \\
\text { Exercise: Wingate test, MVC grip } \\
\text { test, dorsiflexion test (peak power } \\
\text { output) }\end{array}$ & $\begin{array}{l}\text { No significant improvement in } \\
\text { any parameters (force } \\
\text { production, fatigue, power } \\
\text { output) }\end{array}$ \\
\hline Hodges et $a f^{33}$ & $\begin{array}{l}\text { Double-blind RCT } \\
\text { cross-over, single } \\
\text { dose }\end{array}$ & 11 healthy male athletes & $\begin{array}{l}60 \mathrm{mg}, 90 \mathrm{~min} \\
\text { prior to testing }\end{array}$ & $\begin{array}{l}\text { Group A: }(n=11) \\
60 \mathrm{mg} \text { PSE or placebo with } \\
\text { exercise, } 1 \text {-week washout period } \\
\text { ( } 3 \text { trials) } \\
\text { Exercise: } 40 \% \text { submaximal }\end{array}$ & $\begin{array}{l}\text { No significant improvement in } \\
\text { any parameters (peak power, } \\
\text { total work, fatigue, heart rate) }\end{array}$ \\
\hline
\end{tabular}




\begin{tabular}{|c|c|c|c|c|c|}
\hline Study & Design & Subject description & PSE dosage & Main Interventions & Outcome results \\
\hline & & & & $\begin{array}{l}\text { cycling, } 60 \% \text { submaximal cycling, } \\
\text { Wingate test }\left(\mathrm{VO}_{2 p e a k}, \text { peak power }\right. \\
\text { output, gross efficiency) }\end{array}$ & \\
\hline Hodges et $a f^{4}$ & $\begin{array}{l}\text { Double-blind } \\
\text { RCT cross-over, } \\
\text { single dose }\end{array}$ & $\begin{array}{l}\text { Volunteer sample of } 7 \text { male } \\
\text { athletes from a university's } \\
\text { athletic club ( } 1 \text { dropout) }\end{array}$ & $\begin{array}{l}2.5 \mathrm{mg} / \mathrm{kg} \text {, } \\
90 \mathrm{~min} \text { prior to } \\
\text { testing }\end{array}$ & $\begin{array}{l}\text { Group A ( } n=7): 2.5 \mathrm{mg} / \mathrm{kg} \text { PSE or } \\
\text { placebo with exercise, } 2-5 \text {-day } \\
\text { washout period ( } 2 \text { trials) } \\
\text { Exercise: } 1500 \mathrm{~m} \text { time trial (time to } \\
\text { completion, blood parameters) }\end{array}$ & $\begin{array}{l}\text { Significantly decreased time to } \\
\text { completion trial by } 2.1 \% \text { with no } \\
\text { reported side effects }\end{array}$ \\
\hline $\begin{array}{l}\text { Pritchard-Peschek } \\
\text { et } a f^{55}\end{array}$ & $\begin{array}{l}\text { Double-blind } \\
\text { RCT cross-over, } \\
\text { single dose }\end{array}$ & $\begin{array}{l}\text { Volunteer sample of } 6 \text { trained } \\
\text { male cyclists and triathletes }\end{array}$ & $\begin{array}{l}180 \mathrm{mg}, 60 \mathrm{~min} \\
\text { prior to testing }\end{array}$ & $\begin{array}{l}\text { Group A }(n=6) \\
180 \mathrm{mg} \text { PSE or placebo with } \\
\text { exercise, } 2 \text {-week washout period } \\
\text { ( } 2 \text { trials) } \\
\text { Exercise: } 70 \mathrm{~kJ} / \mathrm{kg} \text { standardised } \\
\text { work time trial (time to completion) }\end{array}$ & $\begin{array}{l}\text { Significantly decreased time to } \\
\text { completion by } 5.1 \%\end{array}$ \\
\hline Berry ${ }^{26}$ & $\begin{array}{l}\text { Double-blind } \\
\text { RCT, cross-over, } \\
\text { single dose }\end{array}$ & $\begin{array}{l}\text { Recruited sample of } 13 \text { female } \\
\text { student athletes from Utah Track } \\
\text { and Field ( } 2 \text { dropouts) }\end{array}$ & $\begin{array}{l}2.5 \mathrm{mg} / \mathrm{kg} \text {, } \\
90 \mathrm{~min} \text { prior to } \\
\text { testing }\end{array}$ & $\begin{array}{l}\text { Group A }(n=13) \\
2.5 \mathrm{mg} / \mathrm{kg} \text { PSE or placebo with } \\
\text { exercise, } 1 \text {-week washout period } \\
\text { (2 trials) } \\
\text { Exercise: } 800 \text { m run time trial (time } \\
\text { to completion, heart rate, anxiety } \\
\text { state) }\end{array}$ & $\begin{array}{l}\text { No significant improvement in } \\
\text { any parameters (time to } \\
\text { completion, heart rate, level of } \\
\text { anxiety) }\end{array}$ \\
\hline $\begin{array}{l}\text { Pritchard-Peschek } \\
\text { et } a \Re^{7}\end{array}$ & $\begin{array}{l}\text { Double-blind RCT, } \\
\text { double-blind, } \\
\text { cross-over, multiple } \\
\text { dose }\end{array}$ & $\begin{array}{l}\text { Volunteer sample of } 10 \text { trained } \\
\text { male endurance cyclists from } \\
\text { local cycling/triathlon clubs }\end{array}$ & $\begin{array}{l}2.3 \mathrm{mg} / \mathrm{kg} \text { or } \\
2.8 \mathrm{mg} / \mathrm{kg} \text {, } \\
60 \mathrm{~min} \text { prior to } \\
\text { testing }\end{array}$ & $\begin{array}{l}\text { Group A ( } \mathrm{n}=10) 2.3 \mathrm{mg} / \mathrm{kg}, 2.8 \mathrm{mg} / \\
\mathrm{kg} \text {, or placebo with exercise ( } 3 \\
\text { trials) } \\
\text { Exercise: } 7 \mathrm{~kJ} \mathrm{kg-1BM} \text { work time } \\
\text { trial (time to completion) }\end{array}$ & $\begin{array}{l}\text { No significant improvement in } \\
\text { any parameters (time to } \\
\text { completion) for either dose }\end{array}$ \\
\hline
\end{tabular}




\begin{tabular}{|c|c|c|c|c|c|}
\hline Bias & Gilles et $a^{18}$ & Swain et $a l^{19}$ & Gill et $a^{20}$ & Chester et $a^{21}$ & Chu et $a P^{2}$ \\
\hline $\begin{array}{l}\text { Random sequence } \\
\text { generation (selection bias) }\end{array}$ & $\begin{array}{l}\text { Low risk } \\
\text { 'randomly assigned' }\end{array}$ & $\begin{array}{l}\text { Low risk } \\
\text { 'randomised using block } \\
\text { scheme' }\end{array}$ & $\begin{array}{l}\text { Low risk } \\
\text { 'randomly assigned' }\end{array}$ & $\begin{array}{l}\text { Low risk } \\
\text { 'Latin square design for subject } \\
\text { assignment' }\end{array}$ & $\begin{array}{l}\text { Low risk } \\
\text { 'randomised' }\end{array}$ \\
\hline Allocation concealment & Unclear risk & Unclear risk & Unclear risk & Unclear risk & Unclear risk \\
\hline (selection bias) & Not addressed & Not addressed & Not addressed & Not addressed & Not addressed \\
\hline $\begin{array}{l}\text { Blinding (performance bias } \\
\text { and detection bias) } \\
\text { All outcomes-patients? }\end{array}$ & $\begin{array}{l}\text { Low risk } \\
\text { 'double-blind' }\end{array}$ & $\begin{array}{l}\text { Low risk } \\
\text { 'double-blind' }\end{array}$ & $\begin{array}{l}\text { Low risk } \\
\text { 'double-blind' }\end{array}$ & $\begin{array}{l}\text { Low risk } \\
\text { 'double-blind' }\end{array}$ & $\begin{array}{l}\text { Low risk } \\
\text { 'double-blind' }\end{array}$ \\
\hline $\begin{array}{l}\text { Blinding (performance bias } \\
\text { and detection bias) } \\
\text { All outcomes-providers? }\end{array}$ & $\begin{array}{l}\text { Low risk } \\
\text { 'double-blind' }\end{array}$ & $\begin{array}{l}\text { Low risk } \\
\text { 'double-blind' }\end{array}$ & $\begin{array}{l}\text { Low risk } \\
\text { 'condition allocation was carried out } \\
\text { by associate not involved in study' }\end{array}$ & $\begin{array}{l}\text { Low risk } \\
\text { 'double-blind' }\end{array}$ & $\begin{array}{l}\text { Low risk } \\
\text { 'double-blind' }\end{array}$ \\
\hline Blinding (performance bias & Unclear risk & Unclear risk & Low risk & Unclear risk & Unclear risk \\
\hline $\begin{array}{l}\text { and detection bias) } \\
\text { All outcomes-outcome } \\
\text { assessors? }\end{array}$ & Not addressed & Not addressed & 'double-blind' & Not addressed & Not addressed \\
\hline Incomplete outcome data & Low risk & Low risk & Low risk & Low risk & Low risk \\
\hline $\begin{array}{l}\text { (attrition bias) } \\
\text { All outcomes-dropouts? }\end{array}$ & No dropouts & No dropouts & No dropouts & No dropouts & $\begin{array}{l}1 \text { drop out, addressed and } \\
\text { justified }\end{array}$ \\
\hline Incomplete outcome data & Low risk & Low risk & Low risk & Low risk & Low risk \\
\hline $\begin{array}{l}\text { (attrition bias) } \\
\text { All outcomes-ITT } \\
\text { analysis? }\end{array}$ & $\begin{array}{l}\text { All prespecified outcomes } \\
\text { reported }\end{array}$ & $\begin{array}{l}\text { All prespecified outcomes } \\
\text { reported }\end{array}$ & All prespecified outcomes reported & $\begin{array}{l}\text { All prespecified outcomes } \\
\text { reported }\end{array}$ & $\begin{array}{l}\text { All prespecified outcomes } \\
\text { reported }\end{array}$ \\
\hline Selective reporting & Low risk & Low risk & Low risk & Low risk & Low risk \\
\hline (reporting bias) & $\begin{array}{l}\text { All prespecified outcomes } \\
\text { reported }\end{array}$ & $\begin{array}{l}\text { All prespecified outcomes } \\
\text { reported }\end{array}$ & All prespecified outcomes reported & $\begin{array}{l}\text { All prespecified outcomes } \\
\text { reported }\end{array}$ & $\begin{array}{l}\text { All prespecified outcomes } \\
\text { reported }\end{array}$ \\
\hline Similarity of baseline & Low risk & Low risk & Low risk & Low risk & Low risk \\
\hline characteristics? & $\begin{array}{l}\text { No significant differences } \\
\text { among baseline characteristics }\end{array}$ & $\begin{array}{l}\text { No significant differences } \\
\text { among baseline characteristics }\end{array}$ & $\begin{array}{l}\text { No significant differences among } \\
\text { baseline characteristics }\end{array}$ & $\begin{array}{l}\text { No significant differences } \\
\text { among baseline characteristics }\end{array}$ & $\begin{array}{l}\text { No significant differences } \\
\text { among baseline characteristics }\end{array}$ \\
\hline Co-intervention avoided or & Low risk & Low risk & Low risk & Low risk & Low risk \\
\hline similar? & No co-interventions & No co-interventions & $\begin{array}{l}\text { Cross-over with adequate washout } \\
\text { period }\end{array}$ & No co-interventions & $\begin{array}{l}\text { Cross-over with adequate } \\
\text { washout period }\end{array}$ \\
\hline Compliance acceptable? & Low risk & Low risk & Low risk & Low risk & Low risk \\
\hline & $\begin{array}{l}\text { Compliance with interventions } \\
\text { acceptable }\end{array}$ & $\begin{array}{l}\text { Compliance with interventions } \\
\text { acceptable }\end{array}$ & $\begin{array}{l}\text { Compliance with interventions } \\
\text { acceptable }\end{array}$ & $\begin{array}{l}\text { Compliance with interventions } \\
\text { acceptable }\end{array}$ & $\begin{array}{l}\text { Compliance with interventions } \\
\text { acceptable }\end{array}$ \\
\hline Timing outcome & Low risk & Low risk & Low risk & Low risk & Low risk \\
\hline assessments similar? & $\begin{array}{l}\text { Outcomes measured at same } \\
\text { time across groups }\end{array}$ & $\begin{array}{l}\text { Outcomes measured at same } \\
\text { time across groups }\end{array}$ & $\begin{array}{l}\text { Outcomes measured at same time } \\
\text { across groups }\end{array}$ & $\begin{array}{l}\text { Outcomes measured at same } \\
\text { time across groups }\end{array}$ & $\begin{array}{l}\text { Outcomes measured at same } \\
\text { time across groups }\end{array}$ \\
\hline Overall impression & Low risk & Low risk & Low risk & Low risk & Low risk \\
\hline
\end{tabular}


Table 3 Continued

\begin{tabular}{|c|c|c|c|c|c|}
\hline Bias & Hodges et $a f^{3}$ & Hodges et $a P^{4}$ & Pritchard-Peschek et $a P^{5}$ & Berry $^{26}$ & Pritchard-Peschek et $a P^{7}$ \\
\hline $\begin{array}{l}\text { Random sequence } \\
\text { generation (selection bias) } \\
\text { Allocation concealment } \\
\text { (selection bias) }\end{array}$ & $\begin{array}{l}\text { Low risk } \\
\text { 'randomly assigned' } \\
\text { Unclear risk } \\
\text { Not addressed }\end{array}$ & $\begin{array}{l}\text { Low risk } \\
\text { 'randomised' } \\
\text { Unclear risk } \\
\text { Not addressed }\end{array}$ & $\begin{array}{l}\text { Low risk } \\
\text { 'randomly assigned' } \\
\text { Unclear risk } \\
\text { Not addressed }\end{array}$ & $\begin{array}{l}\text { Low risk } \\
\text { 'random trial' } \\
\text { Low risk } \\
\text { 'member placed capsules in } 2 \text { different } \\
\text { envelopes marked A or B, known only to } \\
\text { this member' }\end{array}$ & $\begin{array}{l}\text { Low risk } \\
\text { 'randomised' } \\
\text { Unclear risk } \\
\text { Not addressed }\end{array}$ \\
\hline $\begin{array}{l}\text { Blinding (performance } \\
\text { bias and detection bias) } \\
\text { All outcomes-patients? }\end{array}$ & $\begin{array}{l}\text { Low risk } \\
\text { 'double-blind' }\end{array}$ & $\begin{array}{l}\text { Low risk } \\
\text { 'double-blind' }\end{array}$ & $\begin{array}{l}\text { Low risk } \\
\text { 'double-blind' }\end{array}$ & $\begin{array}{l}\text { Low risk } \\
\text { 'double-blind' }\end{array}$ & $\begin{array}{l}\text { Low risk } \\
\text { 'double-blind' }\end{array}$ \\
\hline $\begin{array}{l}\text { Blinding (performance } \\
\text { bias and detection bias) } \\
\text { All outcomes-providers? }\end{array}$ & $\begin{array}{l}\text { Low risk } \\
\text { 'double-blind' }\end{array}$ & $\begin{array}{l}\text { Low risk } \\
\text { 'double-blind' }\end{array}$ & $\begin{array}{l}\text { Low risk } \\
\text { 'double-blind' }\end{array}$ & $\begin{array}{l}\text { High risk } \\
\text { 'known to member of research team' } \\
\text { assigning the envelopes A or B }\end{array}$ & $\begin{array}{l}\text { Low risk } \\
\text { 'double-blind' }\end{array}$ \\
\hline $\begin{array}{l}\text { Blinding (performance } \\
\text { bias and detection bias) } \\
\text { All outcomes-outcome } \\
\text { assessors? }\end{array}$ & $\begin{array}{l}\text { Unclear risk } \\
\text { Not addressed }\end{array}$ & $\begin{array}{l}\text { Unclear risk } \\
\text { Not addressed }\end{array}$ & $\begin{array}{l}\text { Unclear risk } \\
\text { Not addressed }\end{array}$ & $\begin{array}{l}\text { Low risk } \\
\text { 'double-blind' }\end{array}$ & $\begin{array}{l}\text { Unclear risk } \\
\text { Not addressed }\end{array}$ \\
\hline $\begin{array}{l}\text { Incomplete outcome data } \\
\text { (attrition bias) } \\
\text { All outcomes-drop-outs? }\end{array}$ & $\begin{array}{l}\text { Low risk } \\
\text { No dropouts }\end{array}$ & $\begin{array}{l}\text { High risk } \\
1 \text { out, not addressed nor } \\
\text { justified }\end{array}$ & $\begin{array}{l}\text { Low risk } \\
\text { No dropouts }\end{array}$ & $\begin{array}{l}\text { Low risk } \\
2 \text { dropouts, addressed and justified }\end{array}$ & $\begin{array}{l}\text { Low risk } \\
\text { No dropouts }\end{array}$ \\
\hline Incomplete outcome data & Low risk & Low risk & Low risk & Low risk & Low risk \\
\hline $\begin{array}{l}\text { All outcomes-ITT } \\
\text { analysis? }\end{array}$ & $\begin{array}{l}\text { All prespecified outcomes } \\
\text { reported }\end{array}$ & $\begin{array}{l}\text { All prespecified outcomes } \\
\text { reported }\end{array}$ & $\begin{array}{l}\text { All prespecified outcomes } \\
\text { reported }\end{array}$ & All prespecified outcomes reported & $\begin{array}{l}\text { All prespecified outcomes } \\
\text { reported }\end{array}$ \\
\hline $\begin{array}{l}\text { Selective reporting } \\
\text { (reporting bias) }\end{array}$ & $\begin{array}{l}\text { Low risk } \\
\text { All prespecified outcomes } \\
\text { reported }\end{array}$ & $\begin{array}{l}\text { Low risk } \\
\text { All prespecified outcomes } \\
\text { reported }\end{array}$ & $\begin{array}{l}\text { Low risk } \\
\text { All prespecified outcomes } \\
\text { reported }\end{array}$ & $\begin{array}{l}\text { Low risk } \\
\text { All prespecified outcomes reported }\end{array}$ & $\begin{array}{l}\text { Low risk } \\
\text { All prespecified outcomes } \\
\text { reported }\end{array}$ \\
\hline $\begin{array}{l}\text { Similarity of baseline } \\
\text { characteristics? }\end{array}$ & $\begin{array}{l}\text { Low risk } \\
\text { No significant differences } \\
\text { among baseline } \\
\text { characteristics }\end{array}$ & $\begin{array}{l}\text { Low risk } \\
\text { No significant differences } \\
\text { among baseline } \\
\text { characteristics }\end{array}$ & $\begin{array}{l}\text { Low risk } \\
\text { No significant differences among } \\
\text { baseline characteristics }\end{array}$ & $\begin{array}{l}\text { Low risk } \\
\text { No significant differences among } \\
\text { baseline characteristics }\end{array}$ & $\begin{array}{l}\text { Low risk } \\
\text { No significant differences among } \\
\text { baseline characteristics }\end{array}$ \\
\hline $\begin{array}{l}\text { Co-intervention avoided or } \\
\text { similar? }\end{array}$ & $\begin{array}{l}\text { Low risk } \\
\text { Cross-over with adequate } \\
\text { washout period }\end{array}$ & $\begin{array}{l}\text { Low risk } \\
\text { Cross-over with adequate } \\
\text { washout period }\end{array}$ & $\begin{array}{l}\text { Low risk } \\
\text { Cross-over with adequate } \\
\text { washout period }\end{array}$ & $\begin{array}{l}\text { Low risk } \\
\text { Cross-over with adequate washout period }\end{array}$ & $\begin{array}{l}\text { Low risk } \\
\text { Cross-over with adequate } \\
\text { washout period }\end{array}$ \\
\hline Compliance acceptable? & $\begin{array}{l}\text { Low risk } \\
\text { Compliance with } \\
\text { interventions acceptable }\end{array}$ & $\begin{array}{l}\text { Low risk } \\
\text { Compliance with } \\
\text { interventions acceptable }\end{array}$ & $\begin{array}{l}\text { Low risk } \\
\text { Compliance with interventions } \\
\text { acceptable }\end{array}$ & $\begin{array}{l}\text { Low risk } \\
\text { Compliance with interventions acceptable }\end{array}$ & $\begin{array}{l}\text { Low risk } \\
\text { Compliance with interventions } \\
\text { acceptable }\end{array}$ \\
\hline $\begin{array}{l}\text { Timing outcome } \\
\text { assessments similar? }\end{array}$ & $\begin{array}{l}\text { Low risk } \\
\text { Outcomes measured at } \\
\text { same time across groups }\end{array}$ & $\begin{array}{l}\text { Low risk } \\
\text { Outcomes measured at } \\
\text { same time across groups }\end{array}$ & $\begin{array}{l}\text { Low risk } \\
\text { Outcomes measured at same } \\
\text { time across groups }\end{array}$ & $\begin{array}{l}\text { Low risk } \\
\text { Outcomes measured at same time } \\
\text { across groups }\end{array}$ & $\begin{array}{l}\text { Low risk } \\
\text { Outcomes measured at same } \\
\text { time across groups }\end{array}$ \\
\hline Overall impression & Low risk & Low risk & Low risk & Low risk & Low risk \\
\hline
\end{tabular}




\begin{tabular}{|c|c|c|c|c|c|}
\hline \multirow[b]{2}{*}{ Outcomes } & \multicolumn{3}{|l|}{ Intervention } & \multirow{2}{*}{$\begin{array}{l}\text { Number of } \\
\text { participants (studies) }\end{array}$} & \multirow{2}{*}{$\begin{array}{l}\text { Quality of the } \\
\text { evidence (GRADE) }\end{array}$} \\
\hline & Placebo & PSE, $60-180 \mathrm{mg}, 1-2 \mathrm{mg} / \mathrm{kg}$ & PSE, >180 $\mathrm{mg}$ or $2.5 \mathrm{mg} / \mathrm{kg}$ & & \\
\hline $\begin{array}{l}\text { Time to completion } \\
\text { (timed trial) }\end{array}$ & $\begin{array}{l}\text { No statistically significant } \\
\text { improvement in all studies }{ }^{16} 18 \quad 1924-27\end{array}$ & $\begin{array}{l}\text { No statistically significant } \\
\text { improvement in all studies }{ }^{18} 192627\end{array}$ & $\begin{array}{l}\text { Significantly decreased time } \\
\text { to completion by } 5.1 \%{ }^{25} \text { and } 2.1 \%{ }^{24}\end{array}$ & $64(7)^{16181924-27}$ & $\begin{array}{l}\oplus \oplus \oplus \bigcirc \\
\text { moderate } \\
\text { Limitations } 0 \\
\text { Imprecision } 0 \\
\text { Inconsistency 0 } \\
\text { Indirectness }-1^{\star} \\
\text { Other 0 }\end{array}$ \\
\hline $\begin{array}{l}\text { Wingate test } \\
\text { Peak anaerobic power }\end{array}$ & $\begin{array}{l}\text { No statistically significant } \\
\text { improvement in both studies } 2022\end{array}$ & $\begin{array}{l}\text { No statistically significant } \\
\text { improvement on study } y^{22}\end{array}$ & $1.6 \%$ improvement $(p=0.07)^{20}$ & $41(2)^{2022}$ & $\begin{array}{l}\oplus \oplus \bigcirc \bigcirc \\
\text { low } \\
\text { Limitations } 0 \\
\text { Imprecision }-1 \dagger \\
\text { Inconsistency 0 } \\
\text { Indirectness }-1^{\star} \\
\text { Other } 0\end{array}$ \\
\hline Peak power of maximal cycling & $\begin{array}{l}\text { No statistically significant } \\
\text { improvement in both studies } \\
2023\end{array}$ & $\begin{array}{l}\text { No statistically significant } \\
\text { improvement on study } y^{23}\end{array}$ & Improved peak power $(p<0.01))^{20}$ & $33(2)^{2023}$ & $\begin{array}{l}\oplus \oplus \bigcirc \bigcirc \\
\text { low } \\
\text { Limitations } 0 \\
\text { Imprecision }-1 \dagger \\
\text { Inconsistency } 0 \\
\text { Indirectness }-1^{*} \\
\text { Other } 0\end{array}$ \\
\hline Respiratory function & $\begin{array}{l}\text { No statistically significant } \\
\text { improvement in all studies }{ }^{18-21} 23\end{array}$ & $\begin{array}{l}\text { No statistically significant } \\
\text { improvement in all studies }{ }^{18} 192123\end{array}$ & $\begin{array}{l}\text { Significantly improved } \\
\text { respiratory function } \\
\left(p=0.02, p=0.01 \text { for } F E V_{1} \text { and } F V C\right)^{20}\end{array}$ & $61(5)^{18-2123}$ & $\begin{array}{l}\oplus \oplus \oplus \bigcirc \\
\text { moderate } \\
\text { Limitations 0 } \\
\text { Imprecision 0 } \\
\text { Inconsistency 0 } \\
\text { Indirectness }-1^{\star} \\
\text { Other 0 }\end{array}$ \\
\hline Isometric muscle test & $\begin{array}{l}\text { No statistically significant } \\
\text { improvement in both studies }{ }^{18} 20\end{array}$ & $\begin{array}{l}\text { No statistically significant } \\
\text { improvement in study }{ }^{18}\end{array}$ & $\begin{array}{l}\text { Significantly improved isometric } \\
\text { knee extension }(p<0.03)^{20}\end{array}$ & $32(2)^{1820}$ & $\begin{array}{l}\oplus \oplus \bigcirc \bigcirc \\
\text { low } \\
\text { Limitations } 0 \\
\text { Imprecision }-1 \dagger \\
\text { Inconsistency 0 } \\
\text { Indirectness }-1^{*} \\
\text { Other } 0\end{array}$ \\
\hline
\end{tabular}

GRADE Working Group grades of evidence.

High quality: further research is very unlikely to change our confidence in the estimate of effect.

Moderate quality: further research is likely to have an important impact on our confidence in the estimate of effect and may change the estimate.

Low quality: further research is very likely to have an important impact on our confidence in the estimate of effect and is likely to change the estimate.

Very low quality: we are very uncertain about the estimate.

New findings

- Doses of PSE $>180 \mathrm{mg}$ or $2.5 \mathrm{mg} / \mathrm{kg}$ shows significant improvement in various athletic performance tests compared with control or doses $60-180 \mathrm{mg}, 1-2 \mathrm{mg} / \mathrm{kg}$.

- Both placebo and doses of PSE $<180 \mathrm{mg}$ or $2.5 \mathrm{mg} / \mathrm{kg}$ had no significant improvement in athletic performance.

- Improvement in athletic performance included: decreased time to completion in timed trials, increased peak anaerobic power, increased FEV ${ }_{1}$ and FVC (lung function).

*No direct comparison of therapeutic doses in interventions.

†Small study group.

$\mathrm{FEV}_{1}$, forced expiratory volume in $1 \mathrm{~s}$; FVC, forced vital capacity; PSE, pseudoephedrine. 
instance, though Pritchard-Peschek et $a l^{25}$ and Hodges et $a t^{24}$ showed significant improvements in timed trials using PSE doses $\geq 180 \mathrm{mg}$ or $2.5 \mathrm{mg} / \mathrm{kg}$, the studies could not be meta-analysed as their interventions were different (1500 $\mathrm{run}^{25}$ and $7 \mathrm{~kJ} / \mathrm{kg}$ body mass work ${ }^{24}$ time to completion, respectively). Owing to such heterogeneity, using the qualitative method of synthesising the evidence was more appropriate. However, this method is sensitive to how studies are categorised, as meeting the criterion of a certain level of evidence depends on the number of studies present in a category, methodology and risk of bias.

All studies were assessed to have a low risk of bias (table 3). For their quality of evidence, the studies were downgraded from high level of evidence to moderate, low or very low depending on the presence of limitations in design, indirectness of evidence, inconsistency of results, imprecision of results and probability of publication bias with the Cochrane GRADE scale (table 4). All studies were RCT in design and implementation and had a low likelihood of bias; thus, none of the studies showed limitations in design. The results of all parameters were also consistent based on dose; only PSE doses $\geq 180 \mathrm{mg}$ or $2.5 \mathrm{mg} / \mathrm{kg}$ showed significant results, while placebo and doses below $180 \mathrm{mg}$ or $2.5 \mathrm{mg} / \mathrm{kg}$ did not. However, only two studies measured the parameters of Wingate test, peak power of maximal cycling and isometric muscle test, leading to small sample sizes. We believe that high quality of evidence should be reserved for conclusions in which the likelihood of making an incorrect reference is small, that is, having consistent findings in multiple sampled studies with low risk of bias. Thus, these categories were downgraded from high-to-moderate quality due to their risk of imprecision. Publication bias of the studies was unclear to assess as only published trials were available through literature search. Additionally, none of the studies directly compared the effects of variable therapeutic doses of PSE. The question of whether higher doses of PSE impact athletic performance would have been more directly addressed if studies had two explicit interventions-a high and low therapeutic PSE dose group-and a control placebo group. This would allow direct analysis between the variables and strengthen the studies' quality of evidence. Thus, all evidence was downgraded in quality due to the indirectness.

The approach to summarising the literature has several strengths. We used a comprehensive, librarian-assisted search of multiple databases. Healthcare professionals decided on article relevance and assessed quality. At least two people extracted the data and the principal investigator verified data entry.

The effect of PSE on athletic performance is a highly debated subject in both the medical and athletic fields. The findings of this review are useful for the design and planning of a larger clinical trial that assesses the effect of PSE on performance with a focus on a direct comparison of doses. PSE has been on and off the WADA guidelines for some time, and present evidence does not indisputably support the banning of PSE at a lower dose. Thus, a large-scale study should be conducted to formulate an approach to this question.

\section{AUTHORS' CONCLUSIONS \\ Implications for practice}

The authors of this review conclude that there is moderate evidence suggesting that higher doses of PSE may be more beneficial than inactive placebo pills or lower doses in enhancing athletic performance. Therefore, these findings should be considered throughout the process of developing substance laws in competition. This would provide a more accurate maximum use of PSE to be considered as doping or whether it has a place on the monitoring list. Since PSE is present in over-the-counter decongestants, changes may allow athletes to take appropriate doses for symptomatic relief while taking the necessary precautions to avoid doping allegations and harmful side effects.

\section{Implications for research}

The banning of substances in competition is a highly debated and continually changing field. Therefore, there is need for a large, high-quality RCT to determine the role of dosing of PSE on enhancing athletic performance.

\section{Implications for an updated systematic review}

There is no recent systematic review on the effects of PSE on athletic performance. Thus, a current systematic review on this topic is necessary to summarise the current findings on PSE use and doping regulations.

Contributors JK and AR ran the literature search, analysed and chose the relevant studies, critiqued their methodology and quality of evidence, and formed relevant conclusions. KVT analysed and chose the relevant studies, critiqued their methodology and quality of evidence, and formed relevant conclusions. All authors proofread the paper.

Competing interests None declared.

Provenance and peer review Not commissioned; internally peer reviewed.

Data sharing statement No additional data are available.

Open Access This is an Open Access article distributed in accordance with the Creative Commons Attribution Non Commercial (CC BY-NC 4.0) license, which permits others to distribute, remix, adapt, build upon this work noncommercially, and license their derivative works on different terms, provided the original work is properly cited and the use is non-commercial. See: http:// creativecommons.org/licenses/by-nc/4.0/

\section{REFERENCES}

1. Schneider AJ, Butcher R. An ethical analysis of drug testing. In: W Wilson, E Derse, eds. Doping in elite sport: the politics of drugs in the Olympic Movement. Champaign-Urbana, IL: Human Kinetics, 2001;129-52.

2. Fitch K. Proscribed drugs at the Olympic Games: permitted use and misuse (doping) by athletes. Clin Med 2012;12:257-60.

3. Clarkson PM, Thompson HS. Drugs and sport. Sports Med 1997;24:366-84.

4. Welcome to the List Wada Prohibited List. (n.d.). Retrieved 1 April 2015. http://list.wada-ama.org/ 
5. Thevis M, Sigmund G, Geyer $\mathrm{H}$, et al. Stimulants and doping in sport. Endocrinol Metab Clin North Am 2010;39:89-105.

6. Tokish JM, Kocher MS, Hawkins RJ. Ergogenic aids: a review of basic science, performance, side effects, and status in sports. Am J Sports Med 2004;32:1543-53.

7. Williams AD, Cribb PJ, Cooke MB, et al. The effect of ephedra and caffeine on maximal strength and power in resistance-trained athletes. J Strength Cond Res 2008;22:464-70.

8. Salerno SM, Jackson JL, Berbano EP. Effect of oral pseudoephedrine on blood pressure and heart rate: a meta-analysis. Arch Intern Med 2005;165:1686-94.

9. Guyatt $\mathrm{GH}$, Oxman AD, Montori $\mathrm{V}$, et al. GRADE guidelines: 5. Rating the quality of evidence - publication bias. J Clin Epidemiol 2011;64:1277-82.

10. Higgins JP, Altman DG, Gøtzsche PC, et al. The Cochrane Collaboration's tool for assessing risk of bias in randomised trials. BMJ 2011;343:d5928.

11. Barroso O, Goudreault D, Carbó Banús ML, et al. Determination of urinary concentrations of pseudoephedrine and cathine after therapeutic administration of pseudoephedrine containing medications to healthy subjects: implications for doping contro analysis of these stimulants banned in sport. Drug Test Anal 2012;4:320-9.

12. Bell DG, Jacobs I. Combined caffeine and ephedrine ingestion improves run times of Canadian Forces Warrior Test. Aviat Space Environ Med 1999;70:325-9.

13. Bell DG, Jacobs I, McLellan TM, et al. Thermal regulation in the heat during exercise after caffeine and ephedrine ingestion. Aviat Space Environ Med 1999;70:583-8.

14. Chester N, Mottram DR, Reilly $\mathrm{T}$, et al. Elimination of ephedrines in urine following multiple dosing: the consequences for athletes, in relation to doping control. Br J Clin Pharmacol 2004;57:62-7.

15. Jolley D, Dawson B, Maloney SK, et al. Hydration and urinary pseudoephedrine levels after a simulated team game. Int J Sport Nutr Exerc Metab 2014;24:325-32.
16. Pokrywka A, Tszyrsznic W, Kwiatkowska DJ. Problems of the use of pseudoephedrine by athletes. Int J Sports Med 2009;30:569-72.

17. Spence A, Sim M, Landers $G$, et al. A comparison of caffeine versus pseudoephedrine on cycling time-trial performance. Int J Sport Nutr Exerc Metab 2013;23:507-12.

18. Gillies H, Derman WE, Noakes TD, et al. Pseudoephedrine is without ergogenic effects during prolonged exercise. J Appl Physiol 1996;81:2611-17.

19. Swain RA, Harsha DM, Baenziger J, et al. Do pseudoephedrine or phenylpropanolamine improve maximum oxygen uptake and time to exhaustion? Clin J Sport Med 1997;7:168-73.

20. Gill ND, Shield A, Blazevich AJ, et al. Muscular and cardiorespiratory effects of pseudoephedrine in human athletes. $\mathrm{Br} \mathrm{J}$ Clin Pharmacol 2000;50:205-13.

21. Chester N, Reilly T, Mottram DR. Physiological, subjective and performance effects of pseudoephedrine and phenylpropanolamine during endurance running exercise. Int J Sports Med 2003;24:3-8.

22. Chu KS, Doherty TJ, Parise G, et al. A moderate dose of pseudoephedrine does not alter muscle contraction strength or anaerobic power. Clin J Sport Med 2002:12:387-90.

23. Hodges AN, Lynn BM, Bula JE, et al. Effects of pseudoephedrine on maximal cycling power and submaximal cycling efficiency. Med Sci Sports Exerc 2003;35:1316-19.

24. Hodges K, Hancock S, Currell K, et al. Pseudoephedrine enhances performance in 1500-m runners. Med Sci Sports Exerc 2006;38:329-33.

25. Pritchard-Peschek KR, Jenkins DG, Osborne MA, et al. Pseudoephedrine ingestion and cycling time-trial performance. Int $J$ Sport Nutr Exerc Metab 2010;20:132-8.

26. Berry C. Effect of Pseudoephedrine on 800-Meter Run Times of NCAA Division I Women Athletes. 2011. http://digitalcommons.usu. edu/cgi/viewcontent.cgi?article $=2115 \&$ context=etd

27. Pritchard-Peschek KR, Jenkins DG, Osborne MA, et al. The doseresponse relationship between pseudoephedrine ingestion and exercise performance. J Sci Med Sport 2014;17:531-4. 